\title{
Altered blood pressure responses and normal cardiac phenotype in ACE2-null mice
}

\author{
Susan B. Gurley, ${ }^{1}$ Alicia Allred, ${ }^{1}$ Thu H. Le, ${ }^{1}$ Robert Griffiths, ${ }^{1}$ Lan Mao, ${ }^{2}$ Nisha Philip, ${ }^{3}$ \\ Timothy A. Haystead, ${ }^{3}$ Mary Donoghue, ${ }^{4}$ Roger E. Breitbart, ${ }^{4}$ Susan L. Acton, ${ }^{4}$ \\ Howard A. Rockman, ${ }^{2}$ and Thomas M. Coffman'1
}

\begin{abstract}
1Division of Nephrology and 'Division of Cardiology, Department of Medicine, Duke University and Durham VA Medical Centers, Durham, North Carolina, USA ${ }^{3}$ Department of Pharmacology and Cancer Biology, Duke University Medical Center, Durham, North Carolina, USA. ${ }^{4}$ Department of Cardiovascular Biology, Millennium Pharmaceuticals Inc., Cambridge, Massachusetts, USA
\end{abstract}

\begin{abstract}
The carboxypeptidase ACE2 is a homologue of angiotensin-converting enzyme (ACE). To clarify the physiological roles of ACE2, we generated mice with targeted disruption of the Ace 2 gene. ACE2-deficient mice were viable, fertile, and lacked any gross structural abnormalities. We found normal cardiac dimensions and function in ACE2-deficient animals with mixed or inbred genetic backgrounds. On the C57BL/6 background, ACE2 deficiency was associated with a modest increase in blood pressure, whereas the absence of ACE2 had no effect on baseline blood pressures in 129/SvEv mice. After acute Ang II infusion, plasma concentrations of Ang II increased almost 3-fold higher in ACE2-deficient mice than in controls. In a model of Ang II-dependent hypertension, blood pressures were substantially higher in the ACE2-deficient mice than in WT. Severe hypertension in ACE2-deficient mice was associated with exaggerated accumulation of Ang II in the kidney, as determined by MALDI-TOF mass spectrometry. Although the absence of functional ACE2 causes enhanced susceptibility to Ang II-induced hypertension, we found no evidence for a role of ACE2 in the regulation of cardiac structure or function. Our data suggest that ACE2 is a functional component of the renin-angiotensin system, metabolizing Ang II and thereby contributing to regulation of blood pressure.
\end{abstract}

\section{Introduction}

The renin-angiotensin system (RAS), which is highly conserved through phylogeny, plays a critical role in regulating blood pressure and cardiovascular functions $(1,2)$. In this cascade, the substrate protein angiotensinogen is cleaved by the aspartyl protease renin to form angiotensin I (Ang I), a decapeptide that is apparently devoid of physiological activity. Ang I is further hydrolyzed by angiotensinconverting enzyme (ACE) to form Ang II. This octapeptide is the major mediator of the diverse biological functions of the RAS. Two distinct pharmacological classes of receptors for Ang II, $\mathrm{AT}_{1}$ and $\mathrm{AT}_{2}$, have been identified, cloned, and sequenced (3). Most of the classically recognized actions of Ang II, including vasoconstriction, stimulation of aldosterone release, and antinatriuretic effects in the kidney, are mediated by $\mathrm{AT}_{1}$ receptors (3). The importance of the RAS in hypertension and in the pathogenesis of heart and kidney disease has been clearly documented through a series of clinical trials with $\mathrm{ACE}$ inhibitors and $\mathrm{AT}_{1}$ receptor blockers (4-11).

ACE, which plays a pivotal role in production of Ang II, is an ectoenzyme consisting of transmembrane and catalytic domains (12). There are 2 distinct forms of ACE, somatic and testicular, both generated by alternative splicing of a single gene $(13,14)$. Somatic ACE is expressed on the surface of endothelial cells throughout the body and is particularly abundant in lung, intestine, choroid plexus, and placenta and on brush border membranes in the kidney. Using genome-based strategies, homologues of ACE have been

Nonstandard abbreviations used: $\mathrm{ACE}$, angiotensin-converting enzyme; $\mathrm{AT}_{1}$ receptor, Ang II receptor type 1; $\mathrm{AT}_{2}$ receptor, Ang II receptor type 2; MALDI-TOF, matrixassisted laser desorption ionization time-of-flight; RAS, renin-angiotensin system; TFA, trifluoroacetic acid.

Conflict of interest: M. Donoghue, R.E. Breitbart, and S.L. Acton carried out this work as employees of Millennium Pharmaceuticals Inc.

Citation for this article: J. Clin. Invest. 116:2218-2225 (2006). doi:10.1172/JCI16980 identified (15-18). One of these, ACE2, exhibits more than $40 \%$ identity at the protein level with the catalytic domain of ACE (15, 16). Similar to ACE, ACE2 is expressed on the surface of certain endothelial cell populations. However, compared with the ubiquitous distribution of ACE, the expression pattern of ACE2 is more limited, with the most abundant expression in kidney, followed by heart and testis $(15,16)$. Their substrate specificities also differ; ACE2 hydrolyzes Ang II with high efficiency but has much lower activity against Ang I (15, 16, 19).

ACE2 was originally identified and cloned from a cDNA library prepared from ventricular tissue of a patient with heart failure (15). The high levels of ACE2 expression in the heart and kidney $(15,16)$ suggest a potential role in cardiovascular regulation. To explore the physiological role of ACE2, we studied the effects of targeted disruption of the Ace 2 gene in mice. Our studies indicate that ACE2 plays a nonredundant role in the regulation of blood pressure through its actions to metabolize Ang II. However, in contrast to previous reports (20), we find no evidence for a major contribution of ACE2 to the regulation of cardiac structure and function.

\section{Results}

Generation of ACE2-deficient mice. The exon of the Ace2 gene containing the Zn-binding signature motif, HEMGH, was replaced with a NEO/URA3 cassette to generate ACE2 KO mice (Figure 1, $A$ and $\mathrm{B}$ ). As shown in Figure 1C, 2 ACE2 transcripts of approximately 3 and $4 \mathrm{~kb}$ can be detected in kidneys of WT mice. Neither transcript could be detected in RNA extracted from kidneys of Ace $2^{-/ y}$ animals using either a murine active-site probe (Figure $1 \mathrm{C}$ ) or a probe derived from the $5^{\prime}$ region of the ACE2 CDNA (data not shown). ACE2 protein was likewise undetectable with Western blots of protein extracts from kidneys of Ace $2^{-1 y}$ animals (Figure 2). Thus, mice with targeted disruption of the Ace2 locus lacked ACE2 
A Targeting construct

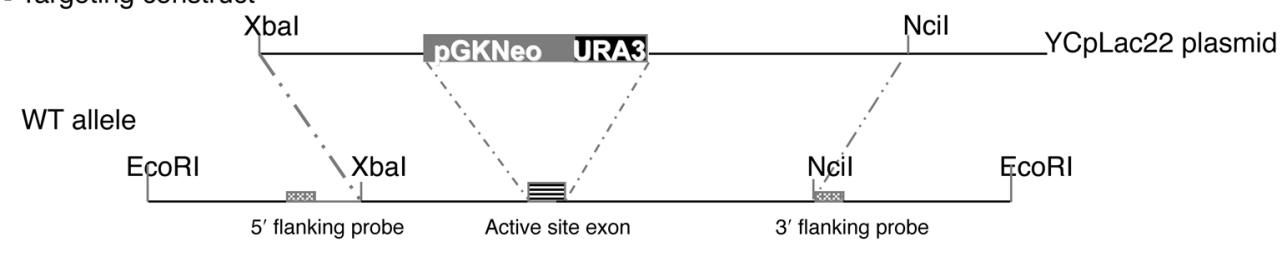

Targeted allele

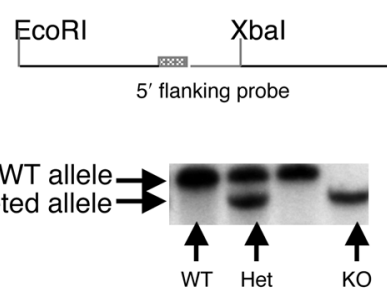

E|coRI

C

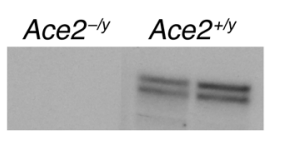

\section{Figure 1}

Generation of ACE2 KO mice. (A) Strategy for producing targeted disruption of the Ace2 gene. In the targeting vector, the exon containing nucleotides +1069 to +1299 encoding the active site of the ACE2 enzyme (including the $\mathrm{Zn}$-binding signature motif, HEMGH) was replaced with a NEO/URA3 cassette. (B) The genotype at the Ace2 locus was determined by Southern blot hybridization using the 5 ' flanking probe. After digestion of genomic DNA by EcoRI, a 6-kb band was diagnostic of the targeted allele, and the WT allele was identified by an 11-kb band. Het, heterozygous. (C) Northern blot analysis of kidneys from Ace2-ly and $A c e 2^{+/ y}$ mice hybridized with a cDNA probe for mouse ACE2. ACE2 mRNA was not detected in kidney RNA from $A c e 2^{-l y}$ mice.

mRNA transcripts and ACE2 protein. The ACE2-null mice were fertile, lacked any gross anatomical or structural abnormalities, and had a normal lifespan.

Echocardiography. To determine the effect of the Ace2 mutation on cardiac function, transthoracic 2-dimensional guided M-mode echocardiography was performed in conscious mice as described previously $(21,22)$. As shown in Table 1 , the echocardiographic findings were virtually identical in the ACE2-deficient animals with mixed genetic background and their WT littermates. This includes similar values for LV end-diastolic and end-systolic dimensions, percentage fractional shortening, and posterior wall thickness. The only significant difference between the groups with mixed genetic background was a modest but significant reduction in heart rate in the Ace $2^{-1 y}$ mice.

As these findings differ from a previous report suggesting that ACE2-deficient mice develop LV dilatation and hypocontractility (20), we backcrossed the Ace2-null mutation for more than 6 generations on each of the parental lines (C57BL/6 and 129/ $\mathrm{SvEv}$ ) and assessed cardiac function by echocardiography in conscious, inbred male C57BL/6- and 129/SvEv-Ace2-/y mice at 3 and 6 months of age. Like the older mice on a mixed genetic background, inbred male C57BL/6- and 129/SvEv-Ace2- $-y$ mice had normal cardiac systolic function compared with their WT littermates at 3 (not shown) and 6 months of age (Table 1). These studies indicate that the absence of functional ACE2 has no detectable effect on cardiac dimensions or ejection fraction in conscious mice under basal conditions.

The contribution of ACE2 to baseline blood pressure regulation. To determine the effects of ACE2 deficiency on regulation of blood pressure, we first measured blood pressures by tail cuff manometry in a group of ACE2-deficient mice with mixed (129/SvEv $\times$ C57BL/6) genetic backgrounds produced within the first few generations after the original germ-line transmission of the Ace 2 mutation from chime- ras (Table 2). In this first cohort of mice, systolic blood pressures were modestly elevated in Ace $2^{-/ y}$ mice $(128 \pm 3 \mathrm{mmHg})$ compared with WT littermates (121 \pm 1 mmHg; $P<0.05)$. By contrast, in a separate cohort of mice with mixed genetic backgrounds generated later after 2 generations of backcrossing onto the 129/SvEv background, we found no difference in systolic blood pressure between the ACE2-deficient mice and their WT littermates $(132 \pm 6$ versus $130 \pm 6 \mathrm{mmHg} ; P=0.83$ ). Because of this variability between blood pressures in distinct groups of animals with mixed genetic backgrounds generated at different stages of backcrossing, we next examined blood pressures in the inbred ACE2-deficient lines.

As shown in Table 2, systolic blood pressures were virtually identical in the $129 / \mathrm{SvEv}$ ACE2-deficient mice and their genetically matched controls $(132 \pm 4$ versus $132 \pm 4 \mathrm{mmHg})$. On the other hand, systolic blood pressures were modestly but significantly increased in C57BL/6 ACE2-deficient mice compared with their WT littermates $(132 \pm 2$ versus $125 \pm 1 \mathrm{mmHg} ; P<0.01)$; the magnitude of the blood pressure increase in these animals $(\sim 7 \mathrm{mmHg})$ was very similar to that observed in the first cohort of mixed-background mice (Table 2). Taken together, these data suggest that the impact of ACE2 on baseline blood pressure homeostasis is strongly influenced by genetic background. A significant contribution of ACE2 to the regulation of blood pressure is apparent only on the permissive C57BL/ 6 background. However, this modest alteration in blood pressure is not sufficient to cause cardiac hypertrophy, as heart weights are similar in C57BL/6 ACE2-deficient and WT mice (heart weight/body weight: $5.0 \pm 0.3$ versus $5.2 \pm 0.7 ; P=0.44)$.

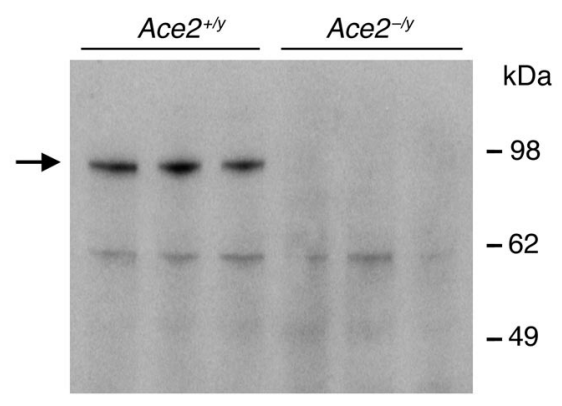

\section{Figure 2}

Western blot of kidney homogenates. Protein extracts were prepared from kidneys of WT and ACE2-deficient mice and size-fractionated on PAGE gels. ACE2 protein was identified with a specific rabbit antiserum. The ACE2 immunoreactive band is absent in ACE2-null animals. 
Table 1

Echocardiography findings in conscious mice

\begin{tabular}{|c|c|c|c|c|c|c|c|c|c|c|}
\hline $\begin{array}{l}\text { Genetic } \\
\text { background }\end{array}$ & Group & $\begin{array}{l}\text { LVDd } \\
(\mathrm{mm})\end{array}$ & $\begin{array}{l}\text { LVDs } \\
\text { (mm) }\end{array}$ & $\begin{array}{l}\text { IVSW } \\
\text { (mm) }\end{array}$ & $\begin{array}{l}\text { PW } \\
(\mathrm{mm})\end{array}$ & $\begin{array}{l}\text { ET } \\
\text { (ms) }\end{array}$ & $\begin{array}{c}\text { HR } \\
\text { (beats/min) }\end{array}$ & $\% F S$ & $\begin{array}{l}\mathrm{LVm} \\
(\mathrm{mg})\end{array}$ & $\mathrm{mVcf}$ \\
\hline \multirow[t]{2}{*}{ Mixed } & $\begin{array}{c}\text { Ace2+/y } \\
(n=9)\end{array}$ & $3.50 \pm 0.05$ & $1.33 \pm 0.04$ & $0.91 \pm 0.03$ & $0.83 \pm 0.03$ & $36.1 \pm 1.1$ & $638 \pm 14$ & $61.8 \pm 0.8$ & $108.6 \pm 4.4$ & $17.4 \pm 0.6$ \\
\hline & $\begin{array}{l}A_{c e 2^{-/ y}} \\
(n=11)\end{array}$ & $3.44 \pm 0.03$ & $1.29 \pm 0.05$ & $0.88 \pm 0.05$ & $0.83 \pm 0.02$ & $37.3 \pm 1.4$ & $611 \pm 16^{A}$ & $62.3 \pm 1.6$ & $103.9 \pm 4.4$ & $17.0 \pm 0.7$ \\
\hline \multirow[t]{2}{*}{ 129/SvEv } & $\begin{array}{l}\text { Ace } 2^{+/ y} \\
(n=10)\end{array}$ & $2.97 \pm 0.07$ & $1.15 \pm 0.06$ & $1.36 \pm 0.05$ & $1.43 \pm 0.07$ & $54.3 \pm 1.5$ & $568 \pm 15$ & $61.6 \pm 1.3$ & $175.4 \pm 8.9$ & $11.5 \pm 0.6$ \\
\hline & $\begin{array}{c}A_{c e 2^{-/ y}} \\
(n=6)\end{array}$ & $2.97 \pm 0.15$ & $1.15 \pm 0.09$ & $1.45 \pm 0.06$ & $1.43 \pm 0.10$ & $54.2 \pm 2.5$ & $564 \pm 27$ & $61.4 \pm 2.0$ & $186.3 \pm 18$ & $11.5 \pm 0.6$ \\
\hline \multirow[t]{2}{*}{ C57BL/6 } & $\begin{array}{l}\text { Ace2 } 2^{+/ y} \\
(n=12)\end{array}$ & $3.14 \pm 0.05$ & $1.32 \pm 0.08$ & $0.90 \pm 0.03$ & $0.98 \pm 0.03$ & $33.5 \pm 0.52$ & $668 \pm 13$ & $58.1 \pm 1.8$ & $102.1 \pm 5.6$ & $17.4 \pm 0.6$ \\
\hline & $\begin{array}{c}A c e 2^{-/ y} \\
(n=8)\end{array}$ & $3.38 \pm 0.09^{A}$ & $1.33 \pm 0.08$ & $0.87 \pm 0.03$ & $0.83 \pm 0.02$ & $33.1 \pm 1.5$ & $657 \pm 13$ & $60.6 \pm 1.4$ & $97.6 \pm 4.7$ & $18.56 \pm 0.9$ \\
\hline
\end{tabular}

AP < 0.05 versus Ace2 ${ }^{+/ y}$. LVDd, LV diastolic diameter; LVDs, LV systolic diameter; IVSW, interventricular septal wall; PW, posterior wall; ET, ejection time; $\mathrm{HR}$, heart rate; \%FS, percentage fractional shortening; LVm, LV mass; mVcf, mean velocity of circumferential fiber shortening; Mixed, $129 / \mathrm{SvEv} \times \mathrm{C} 57 \mathrm{BL} / 6$.

Effects of ACE2 deficiency on plasma levels of Ang II. To examine the effect of the Ace 2 mutation on Ang II metabolism in vivo, we measured plasma Ang II levels before and after Ang II was infused i.v. at $100 \mathrm{ng} / \mathrm{min}$ for 15 minutes. Figure 3 shows that the preinfusion Ang II levels tended to be higher in Ace $2^{-/ y}$ mice than in controls, but this difference was not statistically significant ( $283 \pm 148$ versus $225 \pm 89 \mathrm{fmol} / \mathrm{ml} ; P=0.74)$. After the infusion, serum Ang II levels increased dramatically in both groups and were almost 3 -fold higher in Ace $2^{-/ y}$ mice $(2,644 \pm 454 \mathrm{fmol} / \mathrm{ml})$ than in controls $(918 \pm 370 \mathrm{fmol} / \mathrm{ml} ; P=0.019)$.

Enhanced susceptibility to angiotensin-dependent bypertension in ACE2deficient mice. Because of the apparent differences in acute metabolism of Ang II in the ACE2-deficient mice, we carried out additional experiments to determine whether the absence of ACE2 might enhance the severity of blood pressure elevation in a model of Ang II-dependent hypertension. To this end, we compared the blood pressures of inbred 129/SvEv ACE2-deficient mice with those of 129/SvEv WT controls during chronic infusion of Ang II. For these studies, mice were implanted with radiotelemetry transmitters to continuously measure blood pressures before and during Ang II infusion. After baseline blood pressures were recorded for 7 days, osmotic pumps were implanted to infuse Ang II at $40 \mathrm{pmol} / \mathrm{min}$ for 2 weeks.

During the baseline period, we found that blood pressures were virtually identical in the inbred 129/SvEv ACE2-deficient mice and their WT littermates (24-hour mean arterial pressures: $133 \pm 2$ versus $131 \pm 2 \mathrm{mmHg} ; P=0.65)$. These findings are consistent with the baseline measurements made previously with tail cuff manometry (Table 2). As shown in Figure 4, blood pressures in the WT 129/SvEv mice increased significantly with Ang II infusion $(P=0.0005)$. Over the same period, blood pressures also increased in the ACE2-deficient mice in response to the chronic Ang II infusion $(P=0.0002)$. However, the magnitude of this increase was almost 2-fold greater in the ACE2-deficient animals $(+64 \mathrm{mmHg})$ than in controls (+36 mmHg). Accordingly, after 2 weeks of Ang II infusion, blood pressures were significantly higher in $129 / \mathrm{SvEv}$ Ace $2^{-1 y}$ mice $(195 \pm 6 \mathrm{mmHg})$ than in their WT littermates $(169 \pm 6$ mmHg; $P=0.01$ ) (Figure 4 and Table 3).

Analysis of renal angiotensin peptides. To examine the mechanism for the more marked blood pressure response to Ang II in the
ACE2-deficient mice, we measured levels of Ang II in the kidneys of mice that received chronic infusions of Ang II using matrixassisted laser desorption ionization time-of-flight (MALDI-TOF) mass spectrometry. After chronic Ang II infusion, renal Ang II content increased nearly 6 -fold $(28 \% \pm 5 \%$ versus $5 \% \pm 0.2 \%$ relative intensity; $P=0.0008)$ in the ACE2-deficient mice compared with WT littermates (Figure 5). Thus, the enhanced susceptibility to Ang II-dependent hypertension in the ACE2-deficient mice was associated with marked accumulation of intact Ang II peptide in the kidney.

\section{Discussion}

ACE2 is a recently discovered homologue of ACE that metabolizes angiotensin peptides in vitro with relatively high affinity $(15-17,19,23)$. While ACE2 is expressed in a number of tissues, the kidney is the site of the highest levels of expression, and the pattern of ACE2 expression in the kidney is reminiscent of that of conventional ACE $(15,24-28)$. Moreover, renal expression may be modulated in disease states including diabetic nephropathy (26). There are, however, only limited data on the specific functions of ACE2 in vivo in the intact animal. We have used gene targeting to examine the physiological role of ACE2. Unlike a previous study (20), we find no evidence of a significant role for ACE2 in the modulation of cardiac function. However, our studies identify a role for ACE2 in blood pressure regulation through its capacity to metabolize Ang II in the kidney.

ACE2 expression is relatively abundant in the heart $(15,16)$, and the cDNA for ACE2 was originally identified from a library derived

\section{Table 2}

Systolic blood pressures $(\mathrm{mmHg})$

\begin{tabular}{lcc} 
Genetic background & \multicolumn{1}{c}{ WT } & ACE2 KO \\
Mixed I $(129 / \mathrm{SvEv} \times \mathrm{C} 57 \mathrm{BL} / 6)$ & $121 \pm 1(n=9)$ & $128 \pm 3^{\mathrm{A}}(n=8)$ \\
Mixed II (129/SvEv $\times \mathrm{C} 57 \mathrm{BL} / 6)$ & $130 \pm 6(n=5)$ & $132 \pm 6(n=7)$ \\
129/SvEv & $132 \pm 4(n=10)$ & $132 \pm 4(n=6)$ \\
C57BL/6 & $125 \pm 1(n=19)$ & $132 \pm 2^{\mathrm{B}}(n=15)$
\end{tabular}

${ }^{A} P<0.05$ and ${ }^{B} P<0.01$ versus $W T$. 


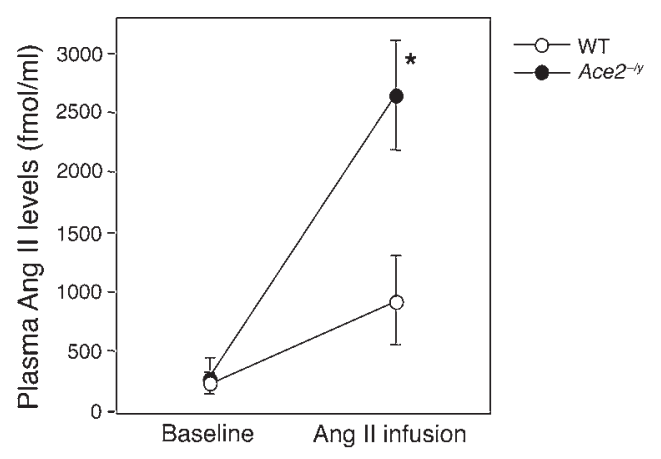

Figure 3

Plasma Ang II levels in $A c e 2^{-/ y}$ and $A c e 2^{+/ y}$ mice. Plasma Ang II levels were measured in $A c 2^{-l y}$ and $A c e 2^{+/ y}$ mice before and after infusion of Ang II. The filled circles depict the ACE2-deficient animals, and the open circles show the WT controls. Data are presented as the mean \pm SEM. ${ }^{*} P=0.019$ versus WT with Ang II infusion.

from ventricular tissue from a patient with congestive heart failure (15). However, our studies would suggest that ACE2 does not play a major role in regulation of normal cardiac function. For example, noninvasive measurements of cardiac function were virtually identical in ACE2-deficient mice and their littermate controls. Specifically, LV end-systolic and end-diastolic dimensions along with percentage fractional shortening were normal in the Ace $2^{-/ y}$ mice of mixed genetic background, and there were no differences between the ACE2-deficient and WT mice in any of the parameters measured.

Our findings differ from a previous report of an independently generated ACE2-deficient mouse line that described a severe cardiac contractility defect as a prominent feature of ACE2 deficiency (20). This phenotype was characterized by increased LV end-diastolic dimension and LV end-systolic dimension, along with reduced ejection fraction; it was more severe in males and became progressively worse with age. This is in marked contrast to our findings of normal cardiac function in ACE2-deficient mice across several different genetic backgrounds and at different ages, including male ACE2-deficient mice that were more than 14 months old (Table 1). The dramatic difference in phenotypes between the 2 ACE2-deficient lines cannot be easily explained by differences in the gene disruption methodology, as the exon encoding the active site of the enzyme was deleted in both lines. One factor that may have impacted the assessments of cardiac function was the use of anesthesia for the echocardiographic measurements. General anesthesia has well-recognized effects to

\section{Figure 4}

Radiotelemetry measurement of systolic blood pressure before and during Ang II infusion. Systolic blood pressures (SBPs) were recorded continuously in WT (open circles) and ACE2 KO (filled circles) mice. Ang II infusion was initiated on day 1. Baseline SBPs were similar in both groups (day 0). Chronic infusion of Ang II caused blood pressures to increase in both groups. However, by day 4 of Ang II infusion, blood pressure was significantly higher in ACE2 KO mice than in controls and remained higher throughout the remainder of the infusion period $\left({ }^{\star} P<0.05\right)$. depress cardiac function (29). Moreover, echocardiographic measurements may be affected substantially by anesthesia, and these effects vary depending on the type and duration of anesthesia (30, 31). Compared with the use of conscious, unanesthetized animals in our studies, it is possible that the use of anesthesia in the previous studies of Crackower et al. might have generated apparent abnormalities in cardiac function (20).

Another common cause of variable phenotypes in transgenic mouse models is a difference in genetic backgrounds. Both lines of ACE2-deficient mice were generated from ES cells derived from 129 strain mice and chimeras that were initially backcrossed to $\mathrm{C} 57 \mathrm{BL} / 6$. The precise genetic background of the animals reported by Crackower et al. (20) was not specified but is likely to have been a random mix of the 2 parental lines. In our studies, there were no apparent cardiac abnormalities in ACE2-deficient animals with mixed genetic backgrounds or in inbred mice generated by separate backcrosses of the Ace 2 mutation onto the 2 original parental backgrounds. Thus, a modifying influence of genetic background does not seem to explain the different phenotypes. Nonetheless, because there was no abnormal cardiac phenotype in our ACE2deficient mice, the line could be used to study the role of ACE2 in regulation of blood pressure without concern for potentially confounding effects of impaired cardiac function.

In separate comparisons of cohorts of mice with mixed or inbred backgrounds, we carefully assessed the effects of ACE2 deficiency on blood pressure. In animals with genetic backgrounds that were a random mix of C57BL/6 and 129, the effect of ACE2 on blood pressure was inconsistent. In one early cohort of mixedbackground mice, blood pressures were higher in ACE2-deficient mice than their WT littermates. However, in a second cohort, backcrossed 2 generations onto the 129/SvEv background, there was no difference in blood pressure between ACE2-deficient and control mice. This variability in blood pressure suggested that there might be genetic modifiers in the parental backgrounds affecting this trait. To test for this possibility, we generated distinct lines of inbred ACE2-deficient mice with homogeneous genetic backgrounds and examined their blood pressures. Blood pressures of inbred 129/SvEv ACE2-deficient mice were virtually identical to those of 129/SvEv controls. By contrast, on the C57BL/ 6 background, ACE2 deficiency was associated with a modest but statistically significant increase in blood pressure of approximately $7 \mathrm{mmHg}$. This level of increase was very similar to that observed in the initial cohort of mixed-background animals. Taken together, these findings suggest that background genes can significantly modify the impact of ACE2 on blood pressure homeostasis. Therefore, in a permissive genetic milieu, ACE2 can contribute significantly to determining the baseline level of blood pressure.

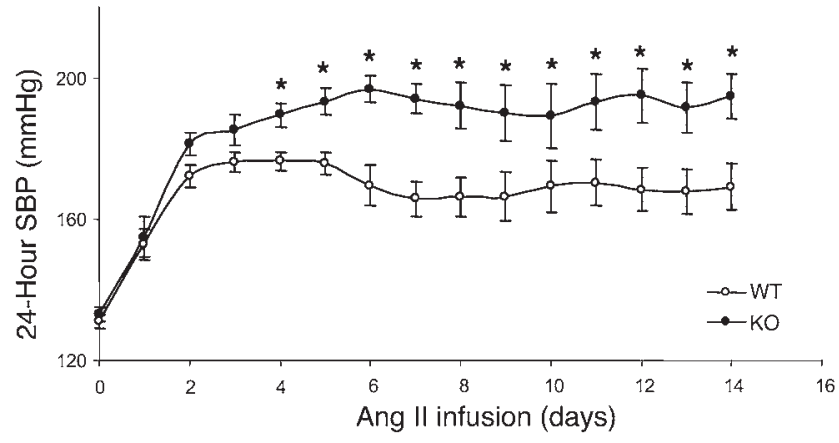


Table 3

Radiotelemetry blood pressure measurements

$\begin{array}{lcc} & \text { WT }(\boldsymbol{n}=\mathbf{9}) & \text { Ace2 }{ }^{-/ y}(\boldsymbol{n}=\mathbf{8}) \\ \text { Baseline SBP }(\mathrm{mmHg}) & & \\ \text { Daytime } & 130 \pm 2 & 126 \pm 3 \\ \text { Nighttime } & 136 \pm 4 & 131 \pm 5 \\ \text { 24-Hour } & 131 \pm 2 & 133 \pm 2 \\ \text { SBP during chronic Ang II infusion (mmHg) } & \\ \text { Daytime } & 163 \pm 7^{\mathrm{A}} & 192 \pm 7^{\mathrm{A}, \mathrm{B}} \\ \text { Nighttime } & 174 \pm 6^{\mathrm{A}} & 198 \pm 5^{\mathrm{A}, \mathrm{B}} \\ \text { 24-Hour } & 169 \pm 6^{\mathrm{A}} & 186 \pm 10^{\mathrm{A}, \mathrm{B}}\end{array}$

AP $<0.006$, paired Student's $t$ test before and after Ang II infusion. B $P<0.03$, unpaired Student's $t$ test between WT and KO.

ACE2 is a mono-carboxypeptidase, and previous in vitro studies have demonstrated its capacity to metabolize angiotensin peptides including Ang I and II $(15,16,19)$. Therefore, we were interested in the effects of ACE2 deficiency on the disposition of plasma Ang II in the intact animal. At baseline, we found no significant differences in plasma Ang II levels between WT and ACE2-deficient mice. However, after an acute i.v. infusion of Ang II, we found that plasma levels of Ang II were almost 3-fold higher in ACE2-deficient mice than in controls. This finding suggests that ACE2 is a key pathway for the metabolism of Ang II in vivo.

In Ang II-dependent hypertension, intrarenal actions of Ang II contribute to antinatriuresis, and elevation of blood pressure is associated with accumulation of Ang II peptide in the kidney (32, 33). Because of the high levels of ACE2 expression in the kidney (15) and our demonstration of the key contribution of ACE2 to Ang II metabolism, we posited that ACE2 might function to attenuate the hypertensive actions of Ang II. To test this hypothesis, we compared the responses of ACE2-deficient and WT mice to chronic infusion of Ang II, a widely used model of angiotensin-dependent hypertension (34). Chronic infusion of 40 $\mathrm{pmol} / \mathrm{min}$ of Ang II over 14 days caused a significant increase in blood pressure in WT mice (Table 3), as expected $(35,36)$. Administration of an equivalent concentration of Ang II to ACE2-deficient mice caused a significantly larger increase in blood pressure that was almost 2 -fold greater than that in controls. Thus, the absence of ACE2 enhances the severity of Ang II-dependent hypertension.

To determine whether the exaggerated severity of hypertension in ACE2-deficient mice was associated with altered metabolism of Ang II in the kidney, we compared the levels of renal angiotensin peptides in the 2 groups using a novel application of MALDI-TOF mass spectrometry. Compared

\section{Figure 5}

Measurement of renal angiotensin peptides using MALDI-TOF mass spectrometry. Representative spectrum for kidney extracts from a WT (A) and an ACE2-deficient mouse (B) after chronic infusion of Ang II. Ang I was added to both samples as an internal standard (IS). The level of Ang II is represented by the peak corresponding to the molecular weight at $1,046.44 \mathrm{~m} / \mathrm{z}$ (massto-charge ratio) in kidney extracts from a WT mouse $(\mathbf{A})$ and an ACE2-deficient mouse (B). Compared with kidneys from WT controls, Ang II was increased nearly 6-fold in kidneys from ACE2-deficient mice. ${ }^{*} P=0.0008$. with other available techniques, this method affords advantages in specificity and sensitivity for angiotensin peptide detection (37, 38). After chronic Ang II infusion, we find that renal Ang II levels are more than 5-fold higher in ACE2-deficient mice than in controls, indicating that the more severe hypertension in ACE2-deficient mice is due to impaired metabolism of Ang II in the kidney. Thus, ACE2 appears to have a critical role to regulate local concentrations of Ang II in the kidney. Because of the powerful effects of Ang II to stimulate renal sodium reabsorption, this represents a potent pathway for controlling blood pressure.

Our findings suggest that ACE2 regulates cardiovascular responses by degrading Ang II and attenuating its actions at the $\mathrm{AT}_{1}$ receptor. However, it should be noted that hydrolysis of Ang II by ACE2 generates another peptide with putative biological actions: angiotensin 1-7 (19). Accumulating evidence suggests that this peptide causes vasodilation and natriuresis and may promote reduced blood pressures (39), and it has been further suggested that ACE2 may be a major pathway for synthesis of angiotensin 1-7 (40). It is therefore conceivable that production of angiotensin 1-7 by ACE2 may have physiological consequences and that reduced synthesis of angiotensin 1-7 might also contribute to the proclivity of ACE2-deficient mice to develop hypertension. However, the robust, 5-fold increase in renal Ang II levels observed in the ACE2-deficient mice is likely sufficient to explain the difference in severity of hypertension, independently of any potential effect of angiotensin 1-7.

In summary, our studies in ACE2-deficient mice have identified a role for ACE2 in regulation of blood pressure, and they establish ACE2 as a functioning component of the RAS in vivo. These findings are consistent with and provide a potential mechanistic basis for the reported linkage of the Ace 2 gene locus with genetic hypertension in rats (20). Our data support a model in which ACE2 contributes to the metabolism of Ang II in vivo, and the absence of this activity results in enhanced susceptibility to
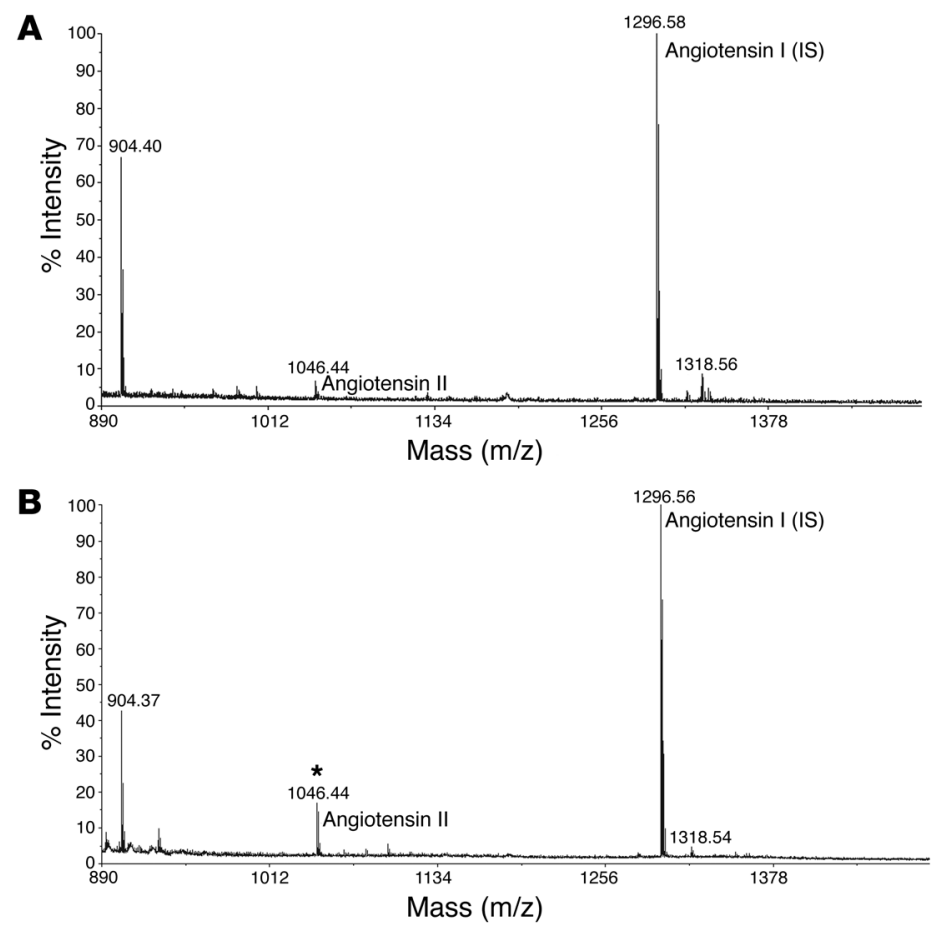
Ang II-dependent hypertension. Because of its very high levels of expression in kidney, we suggest that regulation of blood pressure by ACE2 may primarily involve modulation of the actions of Ang II at renal $\mathrm{AT}_{1}$ receptors.

\section{Methods}

Generation of ACE2-deficient mice. BAC145d21 containing a portion of the murine Ace 2 gene was identified by Southern hybridization using a 1.8-kb EcoRI fragment of the human ACE2 cDNA. A 5.2-kb fragment of BAC $145 \mathrm{~d} 21$ that included the exon containing sequences encoding the active site of the ACE2 enzyme (+1069 to +1299) was subcloned into the yeast shuttle vector YCpLac22 (pMD44). A 3.4-kb fragment containing the NEO/URA3 cassette from PRAY-1 and flanked by Ace2 genomic sequence was generated by PCR and cotransformed with PMD44 into yeast strain YPH501 for homologous recombination. The final linearized targeting construct, shown in Figure 1A, was electroporated into MPI1-12D ES cells that had been derived from 129/SvEvfBRTac mice. G418-resistant ES cells were screened for homologous recombinants by Southern blot hybridization with genomic probes that were outside of the targeting construct (Figure 1, A and B). Targeted ES clones were then injected into C57BL/6H blastocysts to generate chimeras. Male chimeras were crossed with C57BL/6J females, and offspring that received the ES genome were identified by their agouti coat color. Because the ES cell line is XY and the Ace2 gene is located on the $\mathrm{X}$ chromosome, all agouti females were heterozygous for the mutation. These heterozygous females were bred with C57BL/6J male mice and the offspring genotyped by Southern blot hybridization using the 5 ' flanking probe as depicted in Figure 1B.

To document the absence of Ace 2 gene expression, Northern analysis was performed using $10 \mu \mathrm{g}$ total RNA from the kidneys of Ace $2^{-/ y}$ and $A c e 2^{+/ y}$ mice hybridized using a fragment of the mouse cDNA including sequences from the active site of the ACE2 enzyme that should have been deleted by the targeting event and a second probe derived from the $5^{\prime}$ region of the rat ACE2 CDNA.

ACE2-deficient mice were compared with appropriate littermate controls. To determine the effect of genetic background on ACE2 deficiency, inbred animals were generated through backcrossing for at least 6 successive generations onto a C57BL/6J or 129/SvEv genetic background. All mice were bred and maintained in the Association for Assessment and Accreditation of Laboratory Animal Care-accredited animal facilities at Millennium Pharmaceuticals Inc. and the Duke University and Durham VA Medical Centers according to NIH guidelines. Approval for animal care and use in these experiments was granted by the Animal Care and Use Committees of the Duke University and the Durham VA Medical Centers. Analysis of ACE2 protein. Kidneys from Ace $2^{+/ y}$ and Ace2- $-y$ mice were homogenized in $10 \mathrm{mmol} / 1 \mathrm{HEPES}, 150 \mathrm{mmol} / 1 \mathrm{NaCl}, 1 \mathrm{mmol} / 1 \mathrm{EGTA}$, $500 \mathrm{mmol} / \mathrm{MgCl}_{2}$, and $0.02 \% \mathrm{NaN}_{3}, \mathrm{pH} 7.2$, with a protease inhibitor cocktail (Calbiochem) added. Homogenates were centrifuged for $30 \mathrm{~min}$ utes at $1,000 \mathrm{~g}$, and supernatants were stored at $-80^{\circ} \mathrm{C}$. Protein concentration was determined by Bradford assay (Bio-Rad Protein Assay; Bio-Rad Laboratories). For Western analysis of ACE2 protein, rabbit antiserum was generated against human ACE2 peptides 489-508 (EPVPHDETYCDPASLFHVSN) and purified by affinity on a peptide column. The purified antibody, anti-ACE2 ${ }^{(489)}$, was used at a 1:5,000 dilution. Twenty micrograms of the murine kidney homogenates was loaded onto $10 \%$ precast NuPAGE Novex Bis-Tris Gels (Invitrogen), separated at $100 \mathrm{~V}$ for 2 hours, and transferred to Hybond-P (Amersham Biosciences) using a semi-dry transfer system (Hoefer). The blots were incubated in blocking buffer $(5 \%$ nonfat milk, $0.1 \%$ Tween-20, in PBS) for 1 hour at room temperature or overnight at $4{ }^{\circ} \mathrm{C}$. Membranes were then incubated with anti-ACE2 ${ }^{(489)}$ for 1 hour in PBS with $0.1 \%$ Tween-20, 1\% FBS, and were washed once for 15 minutes and 3 times for 5 minutes in PBS containing 0.1\% Tween-20, 1\% FBS. Membranes were incubated with a 1:25,000 dilution of donkey antirabbit-HRP (Amersham Biosciences) in PBS with 0.1\% Tween-20, 1\% FBS for 30 minutes, followed by washing as above and detection using the ECL Plus kit (Amersham Biosciences).

Echocardiography. Transthoracic 2-dimensional guided M-mode echocardiography was performed in conscious mice using an HDI 5000 echocardiograph (Philips) as previously described $(21,22)$. The following parameters were measured: LV end-diastolic dimension (LVEDD); LV end-systolic dimension (LVESD); percentage fractional shortening, calculated as (LVEDD - LVESD)/LVEDD; septal wall thickness; posterior wall thickness; and heart rate-corrected mean velocity of circumferential fiber shortening, calculated as fractional shortening divided by ejection time multiplied by the square root of the R-R interval.

Systolic blood pressure measurements in conscious mice by tail cuff manometry. Systolic blood pressures were measured in conscious mice using a computerized tail cuff system (Visitech Systems) that determines systolic blood pressure with a photoelectric sensor $(41,42)$. This system allows pressures to be measured in 4 mice simultaneously and minimizes the potential for observer bias. Before the study was initiated, mice were adapted to the apparatus for at least 5 days. The validity of this system has been established previously $(41,42)$.

Blood pressure measurements in conscious mice by radiotelemetry. In some experiments, a radiotelemetry system (Transoma Medical) was used to monitor blood pressure in conscious mice, as described previously (43). Mice were anesthetized with isoflurane (44), and a pressure-sensing catheter was implanted in the left carotid artery. The transducer unit was inserted into a subcutaneous pouch generated by blunt dissection inferiorly from a neck incision along the right flank. Mice were allowed 1 week to recover from surgery and regain normal circadian rhythms before measurements were recorded. During the measurement period, mice were housed in a monitoring room where quiet is maintained and no other experiments are performed. Arterial blood pressure measurements from unanesthetized, unrestrained animals were collected, stored, and analyzed using Dataquest ART software (version 3.1; Transoma Medical). Measurements at baseline and during Ang II infusion were taken over a 10-second interval every 5 minutes for at least 7 consecutive days.

Acute infusions of Ang II. In order to examine the role of ACE2 in the metabolism of Ang II in vivo, Ang II peptide was administered by i.v. infusion in WT $(n=5)$ and ACE2-deficient mice $(n=6)$. On the day of study, animals were anesthetized with isoflurane, and a flexible plastic catheter (0.020ID; Braintree Scientific Inc.) was placed in the carotid artery to monitor arterial pressure. A second catheter was placed in the jugular vein to infuse Ang II. After an equilibration period of 5 minutes, a sample of arterial blood was obtained for measurement of plasma Ang II levels as described below. Mice then received an i.v. infusion of $100 \mathrm{ng} / \mathrm{min}$ Ang II over 15 minutes. Ten minutes after the Ang II infusion was completed, a second sample of arterial blood was obtained for measurement of plasma levels of Ang II.

Ang II levels in plasma. Blood was collected into chilled tubes containing a mixed inhibitor solution (0.44 $\mathrm{mM} o$-phenanthrolene, $25 \mathrm{mM}$ EDTA, 1 $\mathrm{mM}$ 4-chloromercuribenzoic acid, and $0.12 \mathrm{mM}$ pepstatin A) as previously described (45). Plasma was obtained by low-speed centrifugation at $4^{\circ} \mathrm{C}$ and applied to AccuBOND II Phenyl Cartridges (Agilent Technologies). Ang II was eluted from the column with methanol, dried, and reconstituted in buffer. Concentrations of Ang II were measured by enzyme immunoassay (46) based on the manufacturer's instructions (SPI bio). With this assay, the lower limit of detectability for Ang II is $1 \mathrm{pg} / \mathrm{ml}$, and cross-reactivity is $4 \%$ for Ang I, 33\% for Ang III, and less than $0.01 \%$ for angiotensin $1-7$.

Chronic Ang II administration. To examine the effects of the Ace2 mutation on susceptibility to Ang II-induced hypertension, Ang II peptide (Sigma- 
Aldrich) was infused chronically. Mice were anesthetized with isoflurane, and ALZET minipumps were implanted subcutaneously. Pumps were preloaded with solutions of Ang II in sterile $0.9 \%$ sodium chloride solution in order to infuse Ang II at a rate of $40 \mathrm{pmol} / \mathrm{min}$ for 28 days. During the infusion, blood pressure was monitored by tail cuff manometry or by radiotelemetry as described above.

Measurement of angiotensin peptides in kidney tissue using MALDI-TOF mass spectrometry. Separate groups of $A c e 2^{+/ y}$ and $A c e 2^{-/ y}$ mice ( $n=10$ per group) received chronic infusions of Ang II as described above, and, after 21 days, Ang II levels in their kidneys were measured. Using an automated tissue homogenizer, kidneys were homogenized in ice-cold methanol and centrifuged at low speed at $4{ }^{\circ} \mathrm{C}$. Supernatants were decanted, dried under a vacuum, and then dissolved in water with $0.1 \%$ trifluoroacetic acid (TFA). Reconstituted extracts were then applied to prepared Sep-Pak solid-phase extraction cartridges (Waters), and eluted using methanol with $0.1 \%$ TFA. Methanol was evaporated, and dried samples were reconstituted in water with $0.1 \%$ TFA. Twenty microliters of kidney extract was dried using vacuum centrifugation to $2 \mu \mathrm{l}$, which was then mixed with $1 \mu \mathrm{l}$ of internal standard (Ang I, $5 \mathrm{pmol}$ ). Three microliters of matrix (50 $\mathrm{mM} \alpha$-cyano-4-hydroxycinnamic acid in 50\% acetonitrile and $0.1 \% \mathrm{TFA}$ ) was added to each sample. One microliter of this mixture was spotted on a target plate and allowed to air-dry. The plate was loaded into an ABI 4700 MALDI TOF/TOF mass spectrometer. The laser voltage was set to $3,800 \mathrm{~V}$, and each data point is a result of 10,000 shots to the sample. Signal intensity ratios of Ang II to the internal standards were used to calculate the relative amount of Ang II in the samples. For the spectra of the standards, 1 pmol each of Ang II, Ang III, and angiotensin 1-7 (Bachem) was spotted with $1 \mu \mathrm{l}$ of matrix and subjected the same treatment as the sample extracts.
Statistics. The values for each parameter within a group are expressed as mean \pm SEM. For comparisons between WT and ACE2-deficient groups, statistical significance was assessed using an unpaired 2-tailed Student's $t$ test. A paired 2-tailed Student's $t$ test was used for comparisons within groups. $P$ values less than 0.05 were considered significant.

\section{Acknowledgments}

We greatly appreciate the expert technical assistance of Chris Best, Vicki Huntress-Fairchild, Don Smith, Brendan McGinn, Mailin Van Hoosear, and Ann Strauss. We also appreciate the expert advice of Dennis Huszar and Lee Varban. The work at Millennium Pharmaceuticals Inc. was part of a collaboration with Eli Lilly and Co. These studies were supported by grants from Millennium Pharmaceuticals Inc. and the NIH (HL56122 and HL49277).

Received for publication September 24, 2002, and accepted in revised form June 6, 2006.

Address correspondence to: Thomas M. Coffman, Building 6/ Nephrology (111I), VA Medical Center, 508 Fulton Street, Durham, North Carolina 27705, USA. Phone: (919) 286-6947; Fax: (919) 286-6879; E-mail: tcoffman@acpub.duke.edu.

R.E. Breitbart's present address is: Departments of Cardiology and Pediatrics, Children's Hospital Boston and Harvard Medical School, Boston, Massachusetts, USA.

S.L. Acton's present address is: Cardium Pharmaceuticals Inc., Cambridge, Massachusetts, USA.
1. Inagami, T. 1994. The renin-angiotensin system. Essays Biochem. 28:147-164.

2. Peach, M. 1977. Renin-angiotensin system: biochemistry and mechanisms of action. Physiol. Rev. 57:313-370.

3. Timmermans, P., et al. 1993. Angiotensin II receptors and angiotensin II receptor antagonists. Pharmacol. Rev. 45:205-251.

4. Brenner, B., et al. 2001. Effects of losartan on renal and cardiovascular outcomes in patients with type 2 diabetes and nephropathy. N. Engl. J. Med. 345:861-869

5. Dahlof, B., et al. 2002. Cardiovascular morbidity and mortality in the Losartan Intervention For Endpoint reduction in hypertension study (LIFE): a randomised trial against atenolol. Lancet. 359:995-1003.

6. [Anonymous]. 1991. Effect of enalapril on survival in patients with reduced left ventricular ejection fractions and congestive heart failure. The SOLVD Investigators. N. Engl. J. Med. 325:293-302.

7. [Anonymous]. 1992. Effect of enalapril on mortality and the development of heart failure in asymptomatic patients with reduced left ventricular ejection fractions. The SOLVD Investigators. N. Engl.J. Med. 327:725-727.

8. Lewis, E., et al. 2001. Renoprotective effect of the angiotensin-receptor antagonist irbesartan in patients with nephropathy due to type 2 diabetes. N. Engl. J. Med. 345:851-860.

9. Lewis, E., Hunsicker, L., Bain, R., and Rohde, R. 1993. The effect of angiotensin-converting-enzyme inhibition on diabetic nephropathy. N. Engl.J. Med. 329:1456-1462.

10. Lindholm, L., et al. 2002. Cardiovascular morbidity and mortality in patients with diabetes in the Losartan Intervention For Endpoint reduction in hypertension study (LIFE): a randomised trial against atenolol. Lancet. 359:1004-1010.
11. Yusuf, S., et al. 2000. Effects of an angiotensin-converting-enzyme inhibitor, ramipril, on cardiovascular events in high-risk patients. The Heart Outcomes Prevention Evaluation Study Investigators. N. Engl. J. Med. 342:145-153.

12. Corvol, P., Williams, T., and Soubrier, F. 1995 Peptidyl dipeptidase A: angiotensin I-converting enzyme. Methods Enzymol. 248:283-305.

13. Ehlers, M., Fox, E., Strydom, D., and Riordan, J. 1989. Molecular cloning of human testicular angiotensin-converting enzyme: the testis isozyme is identical to the $\mathrm{C}$-terminal half of endothelial angiotensin-converting enzyme. Proc. Natl. Acad. Sci. U. S. A. 86:7741-7745.

14. Hubert, C., Houot, A., Corvol, P., and Soubrier, F. 1991. Structure of the angiotensin I-converting enzyme gene. Two alternate promoters correspond to evolutionary steps of a duplicated gene. J. Biol. Chem. 266:15377-15383.

15. Donoghue, M., et al. 2000. A novel ACE-related carboxypeptidase (ACE2) converts angiotensin I to angiotensin1-9. Circ. Res. 87:1-9.

16. Tipnis, S., et al. 2000. A human homolog of angiotensin-converting enzyme. J. Biol. Chem. 275:33238-33243.

17. Turner, A., and Hooper, N. 2002. The angiotensin-converting enzyme gene family: genomics and pharmacology. Trends Pharmacol. Sci. 23:177-183.

18. Zhang, H., et al. 2001. Collectrin, a collecting duct-specific transmembrane glycoprotein, is a novel homolog of ACE2 and is developmentally regulated in embryonic kidneys. J. Biol. Chem. 276:17132-17139.

19. Vickers, C., et al. 2002. Hydrolysis of biological peptides by human angiotensin-converting enzyme-related carboxypeptidase. J. Biol. Chem. 277:14838-14843.

20. Crackower, M., et al. 2002. Angiotensin-converting enzyme 2 is an essential regulator of heart func- tion. Nature, 417:822-828.

21. Esposito, G., et al. 2000. Cellular and functional defects in a mouse model of heart failure. Am. J. Physiol. 279:H3101-H3112.

22. Esposito, G., et al. 2002. Genetic alterations that inhibit in vivo pressure overload hypertrophy prevent cardiac dysfunction despite increased wall stress. Circulation. 105:85-92.

23. Turner, A.J., and Hooper, N.M. 2002. The angiotensin-converting enzyme gene family: genomics and pharmacology. Trends Pharmacol. Sci. 23:177-183.

24. Boehm, M., and Nabel, E.G. 2002. Angiotensinconverting enzyme 2: a new cardiac regulator. N. Engl.J. Med. 347:1795-1797.

25. Lely, A.T., Hamming, I., van Goor, H., and Navis, G.J. 2004. Renal ACE2 expression in human kidney disease. J. Pathol. 204:587-593.

26. Tikellis, C., et al. 2003. Characterization of renal angiotensin-converting enzyme 2 in diabetic nephropathy. Hypertension. 41:392-397.

27. Ye, M., et al. 2004. Increased ACE 2 and decreased ACE protein in renal tubules from diabetic mice: a renoprotective combination? Hypertension. 43:1120-1125.

28. Warner, F.J., et al. 2005. Angiotensin-converting enzyme 2 (ACE2), but not ACE, is preferentially localized to the apical surface of polarized kidney cells. J. Biol. Chem. 280:39353-39362.

29. Vatner, S., and Braunwald, E. 1975. Cardiovascular control mechanisms in the conscious state. N. Engl. J. Med. 293:970-976.

30. Takuma, S., et al. 2001. Anesthetic inhibition in ischemic and nonischemic murine heart: comparison with conscious echocardiographic approach. Am. J. Physiol. 280:H2364-H2370.

31. Roth, D., Swaney, J., Dalton, N., Gilpin, E., and Ross, J. 2002. Impact of anesthesia on cardiac function during echocardiography in mice. Am.J. Physiol. 282:H2134-H2140. 
32. Wang, C.T., Chin, S.Y., and Navar, L.G. 2000. Impairment of pressure-natriuresis and renal autoregulation in ANG II-infused hypertensive rats. Am. J. Physiol. Renal Physiol. 279:F319-F325.

33. Zhuo, J.L., et al. 2002. Ang II accumulation in rat renal endosomes during Ang II-induced hypertension: role of AT(1) receptor. Hypertension. 39:116-121.

34. Mitchell, K., and Navar, L. 1995. Intrarenal actions of angiotensin II in the pathogenesis of experimental hypertension. In Hypertension: pathophysiology, diagnosis and management. J. Laragh and B. Brenner, editors. Raven Press. New York, New York, USA. 1437-1450.

35. Francois, H., Athirakul, K., Mao, L., Rockman, H., and Coffman, T.M. 2004. Role for thromboxane receptors in angiotensin-II-induced hypertension. Hypertension. 43:364-369.

36. Kawada, N., Imai, E., Karber, A., Welch, W.J., and Wilcox, C.S. 2002. A mouse model of angiotensin II slow pressor response: role of oxidative stress.
J. Am. Soc. Nephrol. 13:2860-2868.

37. Desiderio, D.M., et al. 2000. Matrix-assisted laser desorption/ionization mass spectrometric quantification of the mu opioid receptor agonist DAMGO in ovine plasma. J. Mass. Spectrom. 35:725-733.

38. Kokko, K.P., and Dix, T.A. 2002. Monitoring neurotensin[8-13] degradation in human and rat serum utilizing matrix-assisted laser desorption/ionization time-of-flight mass spectrometry. Anal. Biochem. 308:34-41.

39. Ferrario, C., et al. 1991. Angiotensin-(1-7): a new hormone of the angiotensin system. Hypertension. 18(Suppl. 5):III126-III133.

40. Ferrario, C.M., Trask, A.J., and Jessup, J.A. 2005. Advances in biochemical and functional roles of angiotensin-converting enzyme 2 and angiotensin(1-7) in regulation of cardiovascular function. $\mathrm{Am}$. J. Physiol. Heart Circ. Physiol. 289:H2281-H2290.

41. Krege, J., Hodgin, J., Hagaman, J., and Smithies, O. 1995. A computerized system for measuring blood pressure in mice. Hypertension. 25:1111-1115.

42. Ito, M., et al. 1995. Regulation of blood pressure by the type $\mathrm{I}_{\mathrm{A}}$ receptor for angiotensin II. Proc. Natl. Acad. Sci. U. S. A. 92:3521-3525.

43. Crowley, S.D., et al. 2005. Distinct roles for the kidney and systemic tissues in blood pressure regulation by the renin-angiotensin system. J. Clin. Invest. 115:1092-1099. doi:10.1172/JCI200523378.

44. Butz, G.M., and Davisson, R.L. 2001. Long-term telemetric measurement of cardiovascular parameters in awake mice: a physiological genomics tool. Physiol. Genomics. 5:89-97.

45. Allred, A., Chappell, M., Ferrario, C., and Diz, D. 2000. Differential actions of renal ischemic injury on the intra-renal angiotensin system. Am. J. Physiol. 279:F636-F645.

46. Volland, H., et al. 1999. A solid-phase immobilized epitope immunoassay permitting very sensitive and specific measurement of angiotensin II in plasma. J. Immunol. Methods. 228:37-47. 